\title{
Devitrite-based Optical Diffusers
}

Haider Butt, ${ }^{1+}$ Kevin M. Knowles, ${ }^{2}$ Yunuen Montelongo, ${ }^{1}$ Gehan A. J. Amaratunga ${ }^{1}$ and Timothy D. Wilkinson ${ }^{1}$

${ }^{1}$ Electrical Engineering Division, Department of Engineering, University of Cambridge, Cambridge, CB3 OFA, UK

${ }^{2}$ Department of Materials Science and Metallurgy, University of Cambridge, 27 Charles Babbage Road, Cambridge, CB3 OFS, UK

Keywords: devitrite, diffusers, diffraction, nanoscale spacings

\begin{abstract}
Devitrite is a novel material produced by heat treatment of commercial soda-limesilica glass. It consists of fans of needle-like crystals which can extent up to several millimeters and have interspacings of up to few hundred nanometers. To date, only the material properties of devitrite have been reported and there has been a distinct lack of research on using it for optical applications. In this study, we demonstrate that randomly oriented fans of devitrite crystals can act as highly efficient diffusers for visible light. Devitrite crystals produce phase modulation of light because of their relatively high anisotropy. The nanoscale spacings between these needles enable light to be diffused to large scattering angles. Experimentally measured results suggest that light diffusion patterns with beam widths of up to $120^{\circ}$ are produced. Since devitrite is an inexpensive material to produce, it has the potential to be used in a variety of commercial applications.
\end{abstract}


Devitrite, $\mathrm{Na}_{2} \mathrm{Ca}_{3} \mathrm{Si}_{6} \mathrm{O}_{16}$ is the primary crystallisation product formed when commercial sodalime-silica glasses are heat treated for extended periods around $900{ }^{\circ} \mathrm{C} .{ }^{1,2}$ Historically, devitrite used to occur as one of many unwanted crystalline forms found in commercial glassware, ${ }^{3,4}$ but with improvements in commercial manufacturing methods for glasses, interest in devitrite largely disappeared in the scientific literature once it was appreciated how to remove it from the final glass product. For this reason, there does not seem to be any attention given to any technological use of this devitrification product in soda-lime-silica glasses, in contrast to other inorganic glasses in which controlled devitrification has given rise to the family of materials known as glass-ceramics. ${ }^{5}$ Here we present data on the inherent optical scattering properties of fans of needle-like devitrite crystals deliberately formed in soda-lime-silica glass. The nanoscaled needles of devitrite are of the order of the wavelength of visible light. The spacings between the needles are also of the same magnitude, enabling the fans of devitrite to act as highly efficient diffusers for visible light with a $\sim 120^{\circ}$ wide field of view. We suggest that devitrite-based diffusers have the potential for use in a wide range of applications in the optical industry.

The optical properties of devitrite were summarised by Peck. ${ }^{6}$ The three principal refractive indices of this triclinic material are $1.565,1.570$ and 1.580. In deliberate devitrification of sodalime-silica glasses devitrite grows as fans of needles. ${ }^{7}$ The axes of the needles are parallel to the direction along which the refractive index is 1.580 , so that the needles have a positive optical elongation. ${ }^{6}$ 
In this work, we have considered the potential for these fans of needles to act as optical diffusers. $^{8}$ Optical diffusers are widely used in the optical industry for distributing or spreading the light intensity from focussed light sources, producing soft light. The wide range of uses for diffusers vary from optical imaging, photovoltaic, ${ }^{9}$ photolithography, ${ }^{10}$ and in the health industry for producing diffused therapeutic thermal energy. ${ }^{11}$ Visual display systems also require diffusers for increasing the field of view; with the emergence of light emitting diodes (LEDs) as alternative solid state light sources, there is a need for diffusers for spreading concentrated light. $^{12}$ The engineering applications of specific diffusers are dependent on their performance, homogenisation capability and efficiency. ${ }^{8}$

Depending on their application and working principle optical diffuse can diffuse light in one ${ }^{8}$ or two dimensions. ${ }^{13}$ Generally, holographic diffusers are used to achieve two dimensional diffusion of light with the desired patterns or beam widths. ${ }^{14}$ These diffusers are mostly produced using sophisticated and expensive nanofabrication methods for patterning the surfaces which cause the optical diffusion. ${ }^{10,13}$

By comparison, devitrite is an economical material which can be produced at a much larger scale by the simple process of heat treating (devitrifying) soda-lime-silica glass. The devitritebased diffusers will have an advantage when producing two-dimensional diffusion patterns for light with, in addition, a degree of control over the beam width produced. Since it has a similar chemistry to the bulk soda-lime-silica glass, it will have low absorption-related losses, and therefore high transmission efficiency. The high melting point of the glass means that these diffusers can withstand high temperatures, certainly up to the glass transition temperature of the bulk soda-lime-silica glass of over $500{ }^{\circ} \mathrm{C} .{ }^{15}$ An additional advantage that devitrite-based 
diffusers will have over conventional diffusers it that the devitrite crystals giving rise to the optical diffusion grow into the bulk glass and are not simply surface features. Hence, by comparison with optical diffusers relying on the patterning of surfaces, these diffusers are very robust and resistance to damage.

\section{Fabrication}

In this work we have grown devitrite crystals in samples of commercial soda-lime-silica glass through controlled heat treatment. Examples of fans of devitrite crystals nucleated at the surface of the glass block clearly visible in thin sections are shown in the polarised light microscope images in Fig. 1(a-e), with the polariser at $90^{\circ}$ to the analyser, as is usual for such microscopes. The areas containing glass which has not devitrified appear dark under the crossed polarisers, as do needles of devitrite crystals oriented with their axes parallel to either the polariser or analyser. In general, colourful devitrite crystals are viewed at different angular positions within the $30 \mu \mathrm{m}$ thin sections, their colour consistent with the colours expected from Michel-Lévy charts of birefringence. These observations confirm the highly anisotropic nature of the devitrite crystals and that, as a consequence, they change the polarisation of light. ${ }^{6}$ The birefringent behaviour of the devitrite crystals can be further appreciated by inserting a sensitive tint plate into the transmitted light beam at $45^{\circ}$ to the polariser and analyser, as shown in Fig. 1(f). It is evident from Fig. 1 that it is difficult to resolve individual needles of devitrite within the fans. This is because the needles typically have cross-sections of the order of the wavelength of visible light, i.e. a few hundred nanometers. In addition, the spacings between the needles are also of this order. 

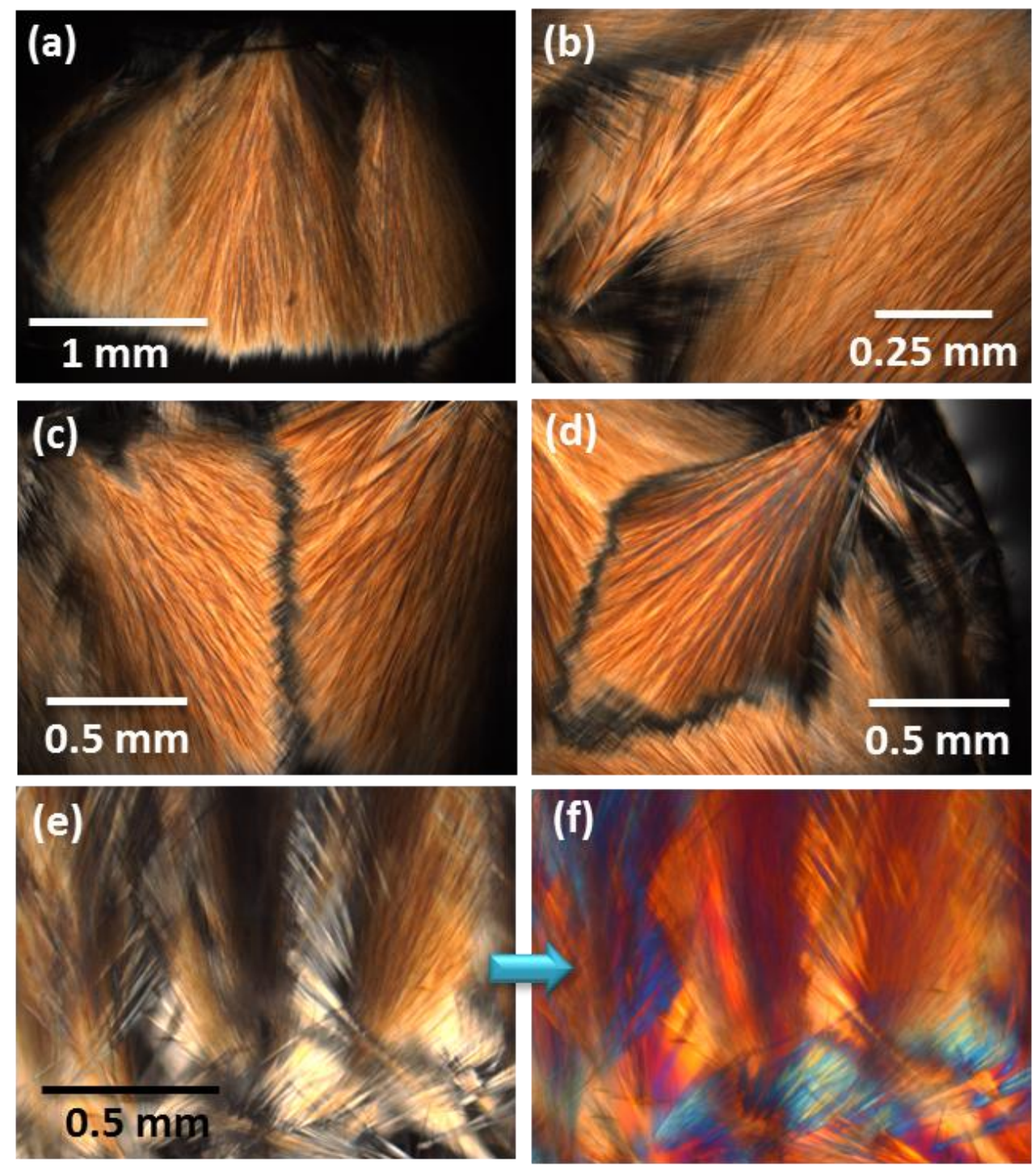

Figure 1 (a-d) Polarised light microscope images showing different arrangements of devitrite crystals within a $30 \mu \mathrm{m}$ thin slice of heat-treated soda-lime-silica float glass. Fans of needle-like crystals are nucleated at the glass surface and then grow into the bulk glass. In three dimensions the fans overlap. (e) An image of several devitrite crystals formed at the edge of the glass, taken with a polarised light microscope. (f) The image of the region in (e) taken with a sensitive tint wave plate inserted at $45^{\circ}$ to the polariser and analyser. 
In three dimensions the fans of devitrite can overlap one another when seen in projection normal to the surface of the $30 \mu \mathrm{m}$ thick sample. This overlapping introduces randomness in the change of polarisation of the light as it is transmitted through the sample. The nanoscale spacings between the needle-shaped crystals enable these crystals to display strong interaction with light and produce optical diffusion. In common with other ceramics and minerals, the refractive indices of devitrite are wavelength-dependent, so that different optical wavelengths undergo different phase retardance. Hence, optical dispersion is observed in devitrite. This is also of technological interest, as it shows that the devitrite crystals could also be used as large area phase modulators for visible light and other optical device applications such as optical holograms. ${ }^{16}$ The size and shape of these fans is dependent on both temperature and time, as well as on the ability for the needles of devitrite to nucleate heterogeneously on the surface of the glass. ${ }^{15}$ As Swift noted in his experiments on the growth of devitrite, heterogeneous nucleation is encouraged by dust $^{7}$ and surface imperfections, while at a particular temperature below the liquidus temperature of the glass the rate of crystal growth is linear until regions of crystallization impinge. For the particular soda-lime-silica float glass used in this work, a crystal growth temperature of $900{ }^{\circ} \mathrm{C}$ was sufficiently near the temperature at which crystal growth was a maximum that we were able to grow long needles of devitrite efficiently in the glass to demonstrate the principle of phase modulation in thin sections. It is clearly possible to control to a high degree the shape and size of the fans of needles by patterning glass surfaces prior to heat treatment and by adjusting the crystal growth temperature. However, such experiments have yet to be undertaken. 


\section{Simulations}

To simulate diffraction patterns from the fans of devitrite crystals, Fast Fourier Transforms (FFTs) of the polarised light microscopy images of devitrite crystals in Fig. 1(a-d) were performed. Different FFT simulations of the far-field diffraction patterns produced from the devitrite crystals in Fig. 1(a-d) by the process of Fraunhofer diffraction are shown in Fig. 2(a-d) respectively. It is evident that the specific arrangement of devitrite crystals in Fig. 1 dictates the diffraction patterns seen in Fig. 2. In accordance with the principle of Fourier Transforms, ${ }^{17}$ the diffraction patterns are always spatially extended (enlarged) in the direction orthogonal to the thin features causing the diffraction. Thus, in Fig. 1(a), the image of the needles of devitrite has a vertical direction relative to which they fan, while its FFT in Fig. 2(a) shows a horizontally extending random distribution of light, with extra intensity along a central horizontal line.

The same is true for other arrangements of devitrite crystals as well, such as in Fig. 1(c), where two fans of devitrite crystals extend diagonally and overlap one other. In the FFT of Fig 1(c) shown in Fig. 2(c), the light is diffused into two directions, giving the appearance of a cross within the intensity distribution in the diffraction pattern. The results confirm that, as expected, the diffusion patterns produced by the devitrite crystals are highly dependent on their directional distribution. In this context, these crystals act as scattering elements towards light, perturbing its normal propagation. The light scattered from the crystals interferes in the far field to produce the diffraction pattern of interest. ${ }^{18}$ If the devitrite crystals were arranged periodically in the form of a lattice, then an order would have also been found in the diffraction patterns. However, as the crystals are almost randomly distributed, they produce diffused diffraction patterns for light. Similar diffraction optics concepts are also used for designing diffraction-based holograms using nanostructures. ${ }^{19}$ At this stage, it is hard to conclude whether the primary reason that devitrite 
crystals cause scattering and consequently the interference of light is the change of phase induced by the crystals or the change in polarisation. It is also possible that both the change of phase and the change in polarisation are required. Further work is needed in this area.
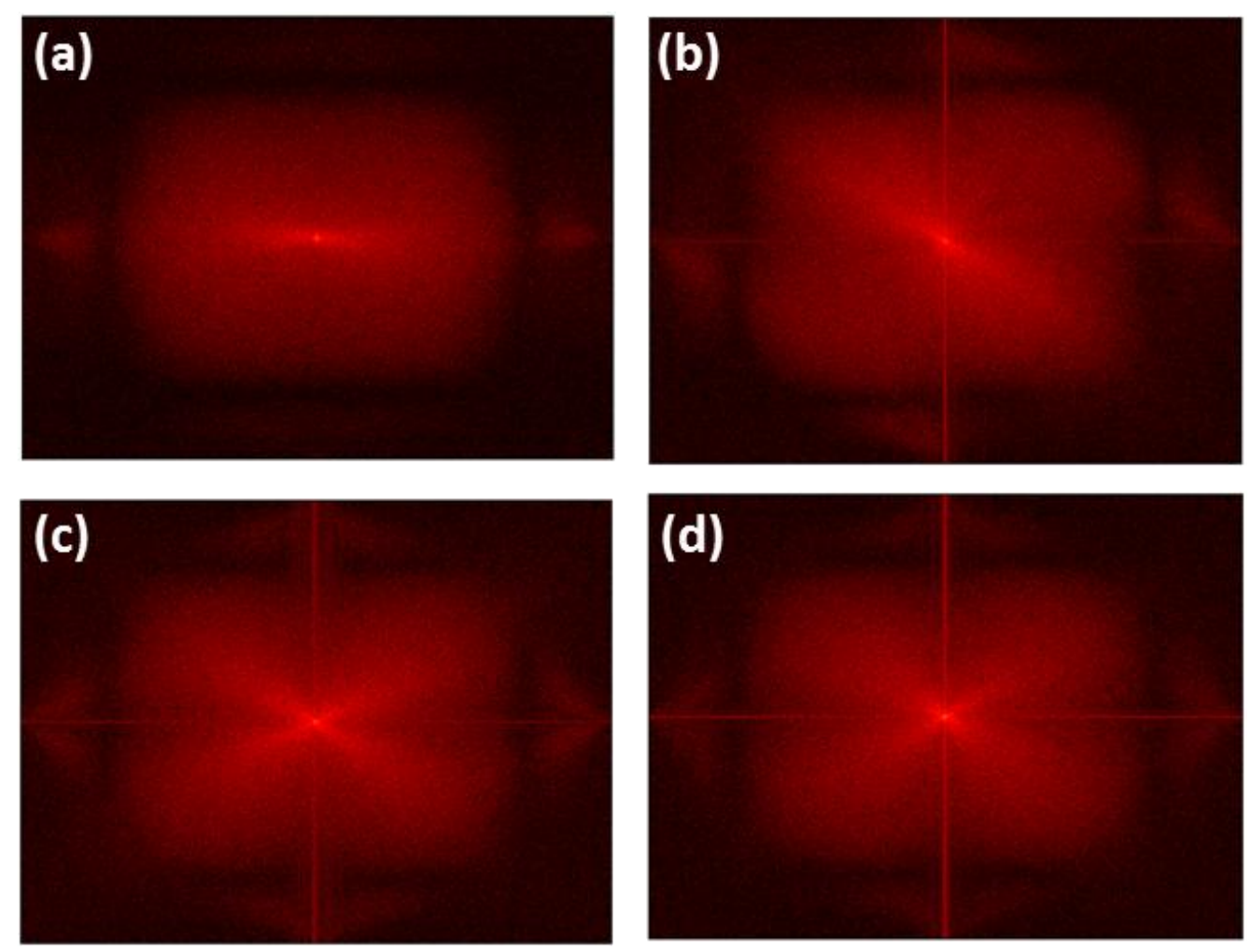

Figure 2 Simulated far-field diffusion patterns for various devitrite crystal arrangements, calculated using FFT. (a-d) 2D FFTs of Fig. 1(a-d) respectively. $\log _{10}$ plots of the FFTs were produced to suppress the zero order intensity and to help make the higher orders in the diffraction patterns more visible. It is evident from the FFTs that the orientation of the devitrite crystals dictates the far field pattern of diffused light.

However, what is clear is that different patterns of diffused light can be obtained by modifying the directional distribution of the devitrite crystals, e.g. either by overlapping different thin sections of devitrified soda-lime--silica glass or choosing parts of a $30 \mu \mathrm{m}$ thin section from 
corners of the original block of glass where overlapping fans of needles arise as a natural consequence of the nucleation and growth process of these fans.

\section{Optical measurements}

To characterise the optical diffusion properties of the thin sections of devitrite experimentally, the thin sections were mounted onto a post with a semi-transparent hemispherical screen set above it. The hemispherical screen had a radius of the order of $15 \mathrm{~cm}$. This allowed sufficient distance from the sample to capture diffraction patterns in the far field. The base of the screen was placed parallel to the plane of the sample as shown in the schematic diagram in Fig. 3(a). The sample was illuminated using a $633 \mathrm{~nm}$ (red) laser. The laser was mounted below the sample, arranged so that the $1 \mathrm{~mm}$ wide beam was normally incident at the sample.

The transmitted laser light was diffracted vertically towards the hemispherical screen. The resultant diffraction patterns produced were captured by a camera. A photograph of the red laser beam passing through bare float glass and then shining on the screen is shown in Fig. 3(b). As expected, the laser beam passes through the glass undistorted and is observed shining on the hemispherical screen and occupying a very small area. The laser source that was placed under the hemispherical screen is also visible because the screen is semi-transparent. The remaining two spots in the figure are artefacts caused by the camera lens when taking the picture. When the same beam is transmitted through the devitrified glass, a strong optical diffusion is observed and the light is spread at large angles (Fig. 3(c-f)). 

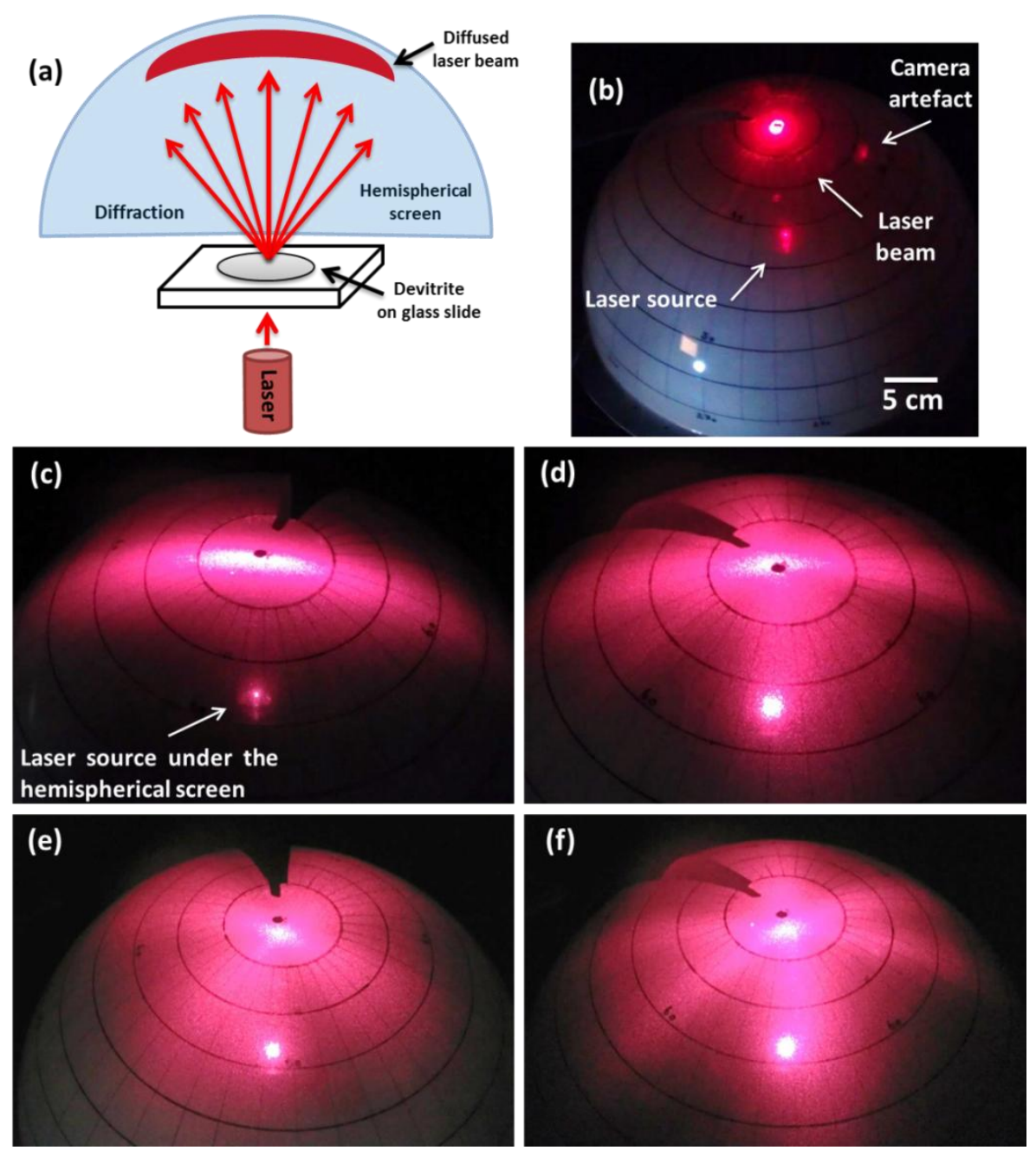

Figure 3 (a) Schematic diagram of the experimental setup used to capture the diffraction patterns from the devitrite crystals. (b) The laser beam passing through bare float glass and illuminating a small area on the hemispherical screen. (c-f) The laser beam passing through different regions of devitrified glass (shown in Fig.1 (a-d) respectively) producing various patterns of optical diffusion. The concentrated laser beam has been diffused to angles as high as $40^{\circ}$, as is evident from the $10^{\circ}$ latitude angular markers on the hemispherical screen. The limitations of the camera used to take this photographs was such that the true extent of the angle over which optical diffusion takes place appears less than the angle seen by the naked eye. 
Fig. 3(c-f) are the experimentally observed diffraction patterns from different arrangements of devitrite crystals shown in Fig. 1 (a-d) respectively and are in excellent agreement with the calculated results from FFT shown in Fig. 2(a-d) respectively. Of particular significance is the extent of the diffusion of the light: up to $40^{\circ}$ symmetrically from the centre.

These results are very encouraging, as they suggest that it is possible to use devitrite, a material which is inexpensive to produce, for applications such as optical diffusers and beam shapers without significant loss in total intensity of the light. Desired beam shapes and intensity distributions can be obtained by using engineered devitrite crystal distributions. Such devitritebased diffusers could also be used in industry for diffusing LED light.

Further experiments were also undertaken to characterise the exact angular distribution (field of view) of light diffused by devitrite samples because of the limitations imposed by the camera used to take the photographs in Fig. 3. A goniometer setup was used to measure the angular distribution of optical intensity, as shown in Fig. 4(a). The devitrite sample was placed in the centre of a goniometer and was then illuminated using a red laser at normal incidence angle. The angular measurements were made on the other side of the sample using a optical power meter mounted on a rotating arm. The rotating arm could be rotated by $180^{\circ}$ around the illuminated sample.

An example of the transmitted light intensity as a function of angle is shown in Fig. 4(b). For this, the region containing unidirectional fans of devitrite crystals (as in Fig. 1(a)) was illuminated, so that the resulting diffused pattern was also distributed mostly in one dimension, as shown in the inset of Fig.4 (b). It can be seen that the devitrite crystals are distributing the intensity of light to angles up to $60^{\circ}$ from the normal, i.e. to significantly higher angles than is 
apparent from Fig. 3. The other important feature observed is that at $0^{\circ}$ there is no very intense peak present from the zero order beam from the laser. Hence, these data confirm that the fans of devitrite produce a phase modulation of the light efficiently, rather than an amplitude modulation.
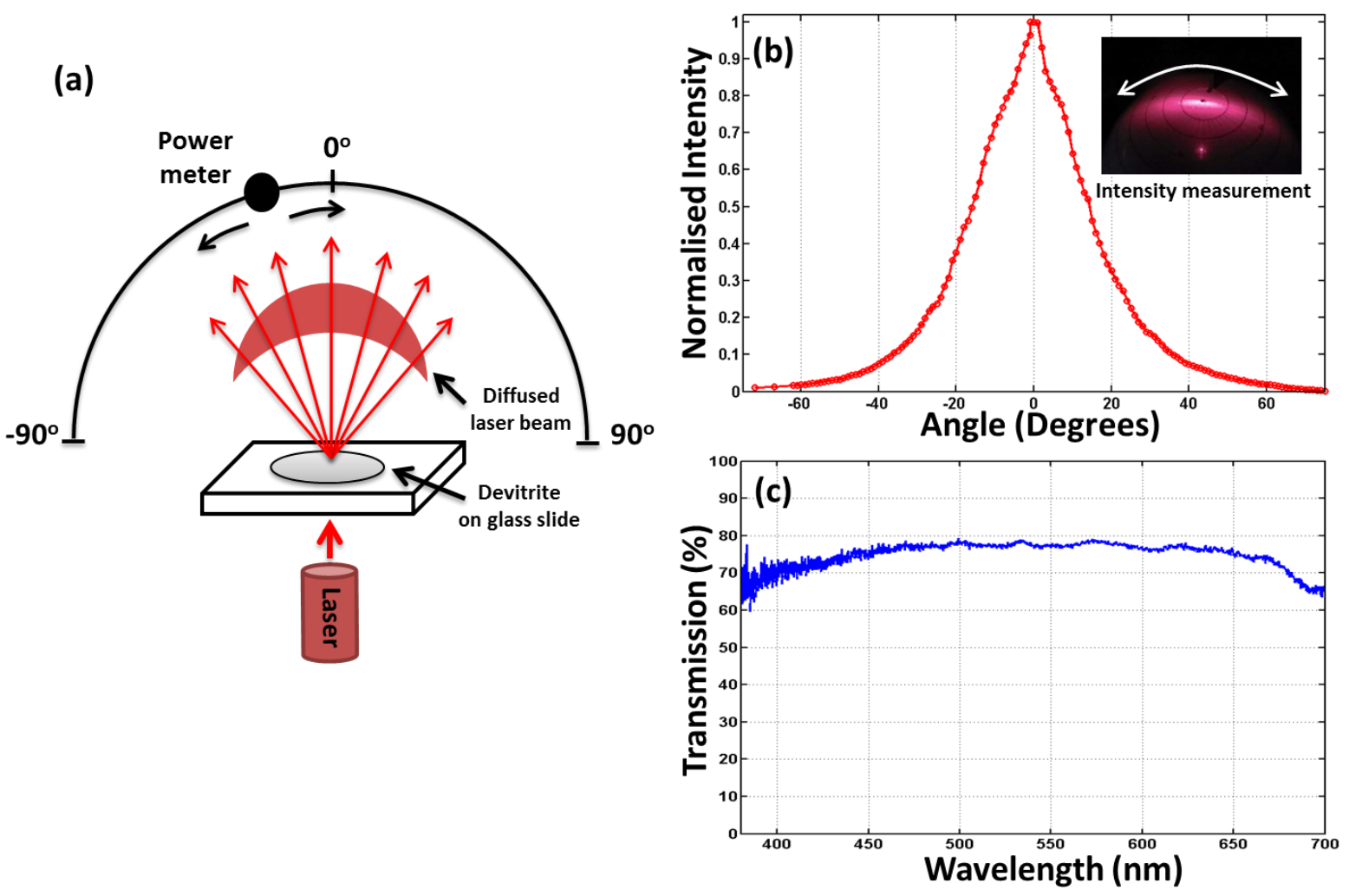

Figure 4 (a) Schematic diagram of the goniometer setup used to measure the angular intensity of the diffused pattern. (b) Transmitted light intensity as a function of angle for the arrangement shown in the inset where a diffuse diffraction pattern is produced from a devitrite region containing a single fan of needles of devitrite. (c) The measured visible transmission through the fan of devitrite. 
For a light beam at normal incidence angle to a periodic grating, the maximum diffraction angle produced can be easily calculated from the equation $\lambda=d \sin \theta$, where $\lambda$ is the wavelength of incident light, $d$ is the spacing between the elements of the gratings, and $\theta$ is the angle between the incident beam and the scattered beam. ${ }^{17}$ For $\theta=60^{\circ}$ and a wavelength $\lambda$ of $633 \mathrm{~nm}$, the value for the minimum spacing $d$ between such grating elements is $730 \mathrm{~nm}$. This figure is consistent with the scale of the spacings between the needle-shaped crystals. It is this fine scale aspect of the microstructure which enables these diffused diffraction patterns with a wide field of view to be produced.

Optical transmission measurements were also conducted on the devitrite samples. The transmission of the visible light was measured using a spectrometer with a resolution of the order of $2 \mathrm{~nm}$ connected to an optical microscope. The devitrite samples were viewed in the transmission mode at $60 \times$ magnification. This allowed the microscope objective to be placed very close to the sample to collect most of the light transmitted through the devitrite. The measurements for unpolarised light were taken at several regions of the devitrite sample and similar results were obtained. The measured average visible transmission through devitrite is shown in Fig. 4(b). The results show that, on average, between the wavelengths of 450 and $650 \mathrm{~nm}$, over $75 \%$ of the light is transmitted through the fans of devitrite crystals - a very encouraging result. In fact, because of the spreading of light by the devitrite crystals to high angles, the results in Fig. 4(b) represent lower bounds on the true transmittance levels because the microscope objective is unable to capture all the light. There are no absorption related losses associated to devitrite, so the $15 \%$ of light not received by the objective is either diffracted at large angles or reflected back. 
Both these measurements were repeated with different linear polarisations of light, but no significant difference was observed. The reason is that there are multiple fans of devitrite crystals with various distributions in the $30 \mu \mathrm{m}$ thick samples of devitrite that we have used. The resulting diffusion patterns cannot be attributed to a single devitrite crystal fan. In future work thinner devitrite samples will be produced, consisting of fewer fans and therefore fewer crystals of devitrite. This will enable a quantitative study to be made of the effect of changing polarisation of light on the diffusion patterns produced and the optical transmission.

Finally, the diffuser performance of devitrite was established for white light. The results obtained are shown in Fig. 5. A white light source was used to illuminate the devitrite sample. The transmitted beam was captured on a flat surface and then recorded on camera. The beam of white light was observed to diffuse noticeably after passing through the sample of devitrite.
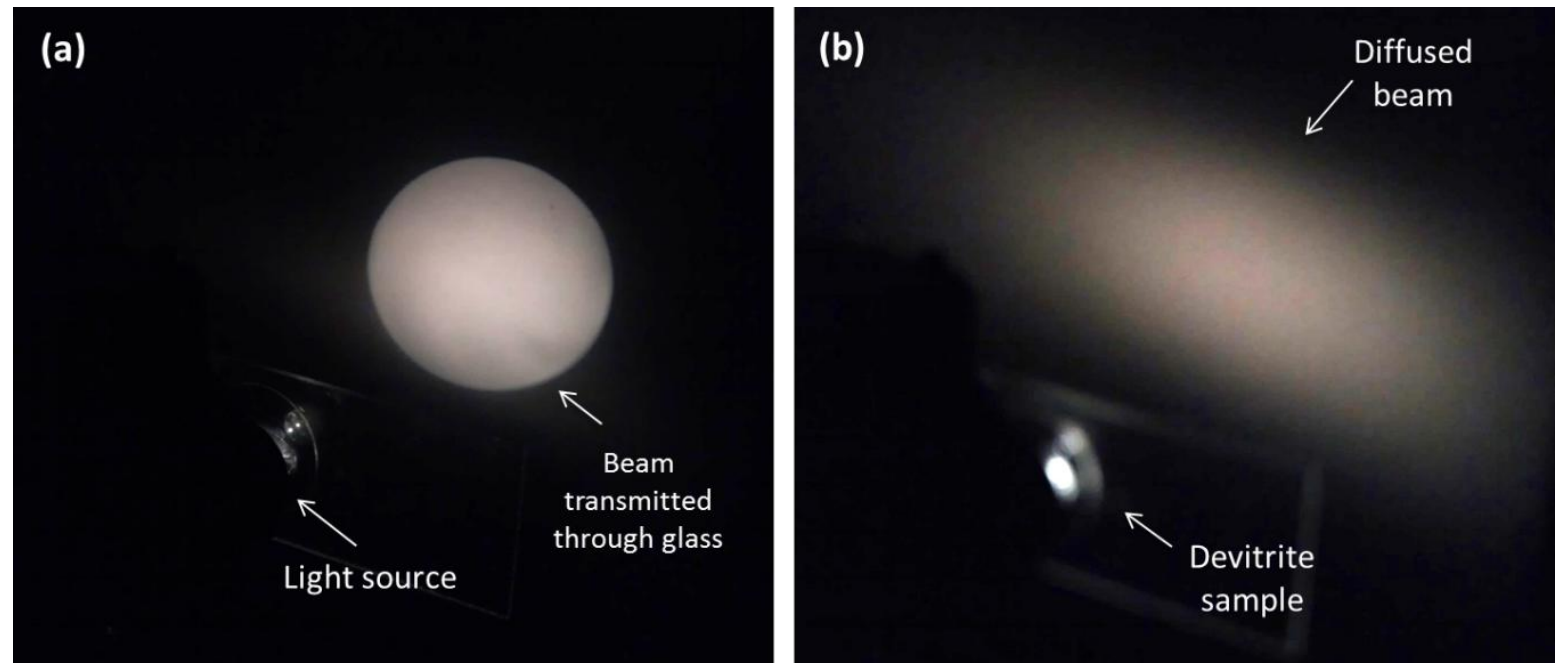

Figure 5 (a) A diverging beam of white light passing through ordinary soda-lime-silica glass.

(b) The same beam after passing through a $30 \mu \mathrm{m}$ thick sample of devitrite. 


\section{Conclusions}

The experiments establish the principle that novel optical diffusers can be produced based on the synthetic material devitrite, $\mathrm{Na}_{2} \mathrm{Ca}_{3} \mathrm{Si}_{6} \mathrm{O}_{16}$. An almost random arrangement of needle-shaped devitrite crystals causes the almost random phase modulation of light and its angular diffusion in the far field. Because of the nanoscale spacing between these needles, the light is diffused to large scattering angles. We suggest that the high efficiency of these diffusers and their inexpensive fabrication process together enable them to have the potential to be used in a variety of applications and industries, such as in medical laser treatments, photonic communication systems, display systems, and for diffusing high power lasers and LEDs.

\section{METHODS SUMMARY}

Devitrite crystals were formed heterogeneously on the surfaces of commercial float glass as a result of prolonged heat treatment. ${ }^{2}$ The crystal growth takes place both parallel to the surface and into the bulk of the glass. The length of the individual devitrite needles of crystals produced

and their concentration depend on the heat treatment time and temperatures used. ${ }^{2,7}$ For the experiments, a $7 \times 10 \times 50 \mathrm{~mm}$ block of float glass was heat treated for $17 \mathrm{hr}$ at $900{ }^{\circ} \mathrm{C}$ to produce individual needles of devitrite up to $2 \mathrm{~mm}$ long. Thin sections were cut transverse to the length of the block for the optical experiments. These were then ground and polished to a standard $30 \mu \mathrm{m}$ thickness using standard mineralogical and petrological specimen preparation methods and subsequently covered with a clear microscope glass cover slip. 
AUTHOR INFORMATION

Corresponding Authors

*h.butt@bham.ac.uk, Tel: +44 121 4158623, Fax: +44 1214143958

\section{Present Addresses}

$\uparrow$ School of Mechanical Engineering, University of Birmingham, Edgbaston, Birmingham B15 2TT, UK

\section{Author Contributions}

YM wrote the software for the FFT simulations and helped in the FFT analysis. GAJA and TDW contributed to the detailed discussion of the experimental data and its significance for industrial applications. HB and KMK wrote the paper which was then approved by the three other coauthors.

\section{Notes}

Due to the high novelty and commercial potential of this invention, a patent for this was filed by Cambridge Enterprise Ltd. in September 2013. A multinational company is also interested in further development of this invention for producing devitrite based LED diffusers.

\section{Acknowledgements}

HB would like to thank The Leverhulme Trust and Cambridge Philosophical Society for research funding. Samples of soda-lime-silica float glass cut to size for the heat treatment experiments were obtained from a commercial glass supplier in Cambridge, UK, Go Glass. 


\section{References}

1. Kahlenberg, V.; Girtler, D.; Arroyabe, E.; Kaindl, R.; Többens, D. M. Devitrite $\left(\mathrm{Na}_{2} \mathrm{Ca}_{3} \mathrm{Si}_{6} \mathrm{O}_{16}\right)$-Structural, Spectroscopic and Computational Investigations on a Crystalline Impurity Phase in Industrial Soda-Lime Glasses. Mineral. Petrol. 2010, 100, $1-9$.

2. Knowles, K. M.; Li, B.; Ramsey, C. N. F.; Thompson, R. P. Microstructural Characterisation of Devitrite, $\mathrm{Na}_{2} \mathrm{Ca}_{3} \mathrm{Si}_{6} \mathrm{O}_{16}$. Adv. Mater. Res. 2012, 585, 51-55.

3. Holland, A. J.; Preston, E. The Microscopical Examination and Identification of Crystalline Products in Commerical Glasses. J. Soc. Glass Technol. 1937, 21, 395-408.

4. Holland, A. J.; Preston, E. The Microscopical Examination and Identification of Crystalline Products in Commerical Glasses. Part II. J. Soc. Glass Technol. 1938, 22, 8298.

5. McMillan, P. W. Glass-Ceramics, 2nd ed.; Academic Press: London, 1979.

6. Peck, A. B. A New Glass Stone: $\mathrm{Na}_{2} \mathrm{O} \cdot 3 \mathrm{CaO} \cdot 6 \mathrm{SiO}_{2}$. J. Am. Ceram. Soc. 1926, 9, 351353.

7. Swift, H. R. Some Experiments on Crystal Growth and Solution in Glasses. J. Am. Ceram. Soc. 1947, 30, 165-169.

8. Bitterli, R.; Scharf, T.; Herzig, H.-P.; Noell, W.; de Rooij, N.; Bich, A.; Roth, S.; Weible, K. J.; Voelkel, R.; Zimmermann, M.; et al. Fabrication and Characterization of Linear Diffusers Based on Concave Micro Lens Arrays. Opt. Express 2010, 18, 14251-14261.

9. Kim, J. M.; Dutta, P. S. Optical Efficiency-Concentration Ratio Trade-off for a Flat Panel Photovoltaic System with Diffuser Type Concentrator. Sol. Energy Mater. Sol. Cells 2012, 103, 35-40.

10. Christophersen, M.; Phlips, B. F. Gray-Tone Lithography Using an Optical Diffuser and a Contact Aligner. Appl. Phys. Lett. 2008, 92, 194102-1-3.

11. Kosoglu, M. A.; Hood, R. L.; Rossmeisl, J. H.; Grant, D. C.; Xu, Y.; Robertson, J. L.; Rylander, M. N.; Rylander, C. G. Fiberoptic Microneedles: Novel Optical Diffusers for Interstitial Delivery of Therapeutic Light. Lasers Surg. Med. 2011, 43, 914-920.

12. Morris, G. M.; Sales, T. R. M. Structured Screens for Controlled Spreading of Light. US Patent 7,033,736, 2006.

13. Jaax, M.; Wolff, S.; Laegel, B.; Fouckhardt, H. Optical and THz Galois Diffusers. J. Eur. Opt. Soc. Rapid Publ. 2013, 8, 13020-1-5.

14. Tedesco, J. M.; Brady, L. A. K.; Colburn, W. S. Holographic Diffuser for Back-Lit Display. US Patent 5,471,327, 1995.

15. Zarzycki, J. Glasses and the Vitreous State; Cambridge University Press: Cambridge, 1991.

16. Butt, H.; Montelongo, Y.; Butler, T.; Rajesekharan, R.; Dai, Q.; Shiva-Reddy, S. G.; Wilkinson, T. D.; Amaratunga, G. A. J. Carbon Nanotube Based High Resolution Holograms. Adv. Mater. 2012, 24, OP331-OP336.

17. Goodman, J. W. Introduction To Fourier Optics, 3rd ed.; Roberts and Company Publishers: Englewood, Colorado, 2005.

18. Butt, H.; Butler, T.; Montelongo, Y.; Rajesekharan, R.; Wilkinson, T. D.; Amaratunga, G. A. J. Continuous Diffraction Patterns from Circular Arrays of Carbon Nanotubes. Appl. Phys. Lett. 2012, 101, 251102-1-4. 
19. Montelongo, Y.; Butt, H.; Butler, T.; Wilkinson, T. D.; Amaratunga, G. A. J. Computer Generated Holograms for Carbon Nanotube Arrays. Nanoscale 2013, 5, 4217-4222. 